\title{
MESA 3: EFECTOS CARDIOVASCULARES DE FÁRMACOS ANTIDIABÉTICOS
}

\section{TABLE 3: CARDIOVASCULAR EFFECTS OF ANTI-DIABETIC DRUGS}

\author{
Coordinadores: Pablo Javier Avila ${ }^{1}$, Silvana Milrad ${ }^{2}$, Mariano Forlino ${ }^{3}$ \\ Secretaria: Sofía Fabregues ${ }^{4}$ \\ Experta invitada: Fabiana Vázquez ${ }^{5}$ \\ Integrantes: Cristina Grosso ${ }^{6}$, Ana Schindler ${ }^{7}$, Fabio Zambon ${ }^{8}$, María Virginia Rodríguez ${ }^{9}$, \\ Natalia Carolina Garrido Santos ${ }^{10}$, Laura Calvo ${ }^{11}$, Alejandra Maldini ${ }^{12}$, Sandra Maserati ${ }^{13}$, Romina Stagno ${ }^{14}$
}

\section{RESUMEN}

Los eventos cardiovasculares representan la mayor complicación de la diabetes. La evidencia sugiere que la metformina mejora los resultados cardiovasculares en pacientes con diabetes, especialmente en el United Kingdom Prospective Diabetes Study (UKPDS) y otros estudios posteriores, por distintos mecanismos. Hay pocos estudios de seguridad cardiovascular para sulfonilureas aunque no tendrían un perfil seguro a este nivel. La gliclazida parece ser la de mejor performance de las drogas de este grupo. Algo similar ocurre con las meglitinidas, para las cuales los datos indican que no aumentarían el riesgo pero tampoco mejorarían la incidencia de eventos cardiovasculares.

Las tiazolidinedionas son las drogas más cuestionadas, aunque los estudios y metaanálisis son contradictorios no habría dudas que aumentan el riesgo de insuficiencia cardíaca. Los inhibidores de la DPPIV mostraron resultados neutros a excepción de saxagliptina que aumentaría el riesgo de internación por insuficiencia cardíaca. Existen datos convincentes que los inhibidores de los receptores SGLT-2 a nivel renal y los análogos del GLP-1 intestinal tienen efectos positivos a nivel cardiovascular, con algunas diferencias entre los integrantes de esta familia.

En cuanto a las insulinas, los estudios sugieren que tanto los análogos lentos como rápidos tendrían un mejor perfil cardiovascular, ligado principalmente a la menor incidencia de hipoglucemias severas, que insulina NPH y regular respectivamente.

Palabras clave: agentes antidiabéticos; diabetes mellitus; eventos cardiovasculares; estudios de seguridad cardiovascular; antidiabéticos orales; insulina.

Revista de la Sociedad Argentina de Diabetes 2020; Vol. 54 (107-122)

\section{ABSTRACT}

Cardiovascular events represent the greatest complication of diabetes. Evidence suggests that metformin improves $\mathrm{CV}$ outcomes in patients with diabetes, especially in the United Kingdom Prospective Diabetes Study (UKPDS) and other subsequent studies, by different mechanisms.

There are few cardiovascular safety studies for sulfonylureas although they would not have a safe profile at this level. Gliclazide appears to be the best performing drug in this group. Something similar occurs with meglitinides for which the data indicates that they would not increase the risk but neither would they improve the incidence of cardiovascular events. Thiazolidinediones are the most questioned drugs, although the studies and meta-analyzes are contradictory, there would be no doubt that they increase the risk of heart failure. DPPIV inhibitors showed neutral results except for saxagliptin, which would increase the risk of hospitalization for heart failure.

There is convincing data that SGLT-2 receptor inhibitors at the renal level and intestinal GLP-1 analogues have positive effects at the cardiovascular level with some differences between the members of these families.

Regarding insulins, studies suggest that both slow and fast analogues would have a better cardiovascular profile, mainly linked to the lower incidence of severe hypoglycemia, than $\mathrm{NPH}$ and regular insulin, respectively.

Key words: antidiabetic agents; diabetes mellitus; cardiovascular events; cardiovascular outcome trial; oral antidiabetics; insulin.

Revista de la Sociedad Argentina de Diabetes 2020; Vol. 54 (107-122)
Coordinador del Comité de Graduados, Sociedad Argentina de Diabetes (SAD), Médico de la Obra Social de Empleados Públicos, San Rafael, Mendoza, Argentina

2 Secretaria del Comité de Hipertensión y otros Factores de Riesgo Cardiovascular, Sociedad Argentina de Diabetes (SAD), Jefa a cargo del Área de Urgencias y Diabetología, Hospital de Rehabilitación Manuel Rocca, Gobierno de la Ciudad de Buenos Aires (GCBA), Ciudad Autónoma de Buenos Aires, Argentina

3 Miembro del Comité de Graduados, Sociedad Argentina de Diabetes (SAD), Director Médico del Centro Médico IMED, San Luis, Argentina

4 Miembro del Comité de Graduados de la Sociedad Argentina de Diabetes (SAD), Médica de Planta, Centro de Salud y Acción Comunitario, Nivel 1, Balvanera (CeSAC No 11), Ciudad Autónoma de Buenos Aires, Argentina
Miembro del Comité de Hipertensión Arterial y otros Factores de Riesgo Cardiovascular, Sociedad Argentina de Diabetes (SAD), Médica de Planta del Servicio de Endocrinología, Metabolismo, Nutrición y Diabetes, Hospital Británico de Buenos Aires, Ciudad Autónoma de Buenos Aires, Argentina

6 Miembro del Comité de Hipertensión Arterial y otros Factores de Riesgo Cardiovascular, Sociedad Argentina de Diabetes (SAD), Médica de Planta, Servicio de Clínica Médica, Hospital Municipal de Vicente López, Provincia de Buenos Aires, Argentina

7 Miembro del Comité de Graduados, Sociedad Argentina de Diabetes (SAD), Médica de Planta, Centro de Salud y Acción Comunitario Nivel 1 (CeSAC) N¹5, Ciudad Autónoma de Buenos Aires, Argentina 
Revista de la Sociedad Argentina de Diabetes Vol. 54 № 2 Suplemento I Jornadas Conjuntas del Comité de Graduados y el Comité de Hipertensión Arterial y otros FRCV. XVIII Jornadas del Comité de Graduados

Mayo-agosto de 2020: 107-122 ISSN 0325-5247 (impresa) ISSN 2346-9420 (en línea)

8 Miembro del Comité de Hipertensión Arterial y otros FRCV, Sociedad Argentina de Diabetes (SAD), Gerente de Economía de la Salud, Laboratorio Novo Nordisk, Ciudad Autónoma de Buenos Aires, Argentina

9 Miembro del Comité de Graduados, Sociedad Argentina de Diabetes (SAD), Asesora Médica, Laboratorio Novo Nordisk, Ciudad Autónoma de Buenos Aires, Argentina

10 Miembro del Comité de Graduados, Sociedad Argentina de Diabetes (SAD), Medical Scientific Liason, Laboratorio Novo Nordisk, Ciudad Autónoma de Buenos Aires, Argentina

11 Miembro del Comité de Graduados, Sociedad Argentina de Diabetes (SAD), Médica de Planta, Clínica Médica, Hospital Independencia, Santiago del Estero, Argentina

12 Miembro del Comité de Graduados, Sociedad Argentina de Diabetes (SAD), Médica Diabetóloga, Centro de Salud Santa Rosa, Universidad Nacional de La Pampa, La Pampa, Argentina

13 Miembro del Comité de Hipertensión Arterial y otros Factores de Riesgo Cardiovascular, Sociedad Argentina de Diabetes (SAD), Médica Diabetóloga de Planta, Centro de Diabetes de Pilar, Municipalidad de Pilar, Provincia de Buenos Aires, Argentina
14 Miembro del Comité de Graduados, Sociedad Argentina de Diabetes (SAD), Médica de Planta, Servicio Clínica Médica, Hospital Municipal de Colón y Hospital Municipal de Chacabuco, Provincia de Buenos Aires, Argentina
Contacto del autor: Pablo Javier Avila
E-mail: drpabloavila@gmail.com
Correspondencia: Bombal 333, San Rafael, CP5600, Men- doza, Argentina
Fecha de trabajo recibido: 09/07/20
Fecha de trabajo aceptado: 03/08/20
Conflictos de interés: no presentan conflictos de interés con el tema de esta mesa de actualización y su publicación los Dres. Pablo Javier Avila, Silvana Milrad, Mariano Forli- no, Sofía Fabregues, Cristina Grosso, Ana Schindler, Laura Calvo, Alejandra Maldini, Sandra Maserati y Romina Stag- no. La Dra. Fabiana Vázquez recibió honorarios como di- sertante de: Boheringer Ingelheim, Novo Nordisk, Eli Lilly, Sanofi Aventis, AstraZeneca, Servier, TEVA, Merck Sharp \& Dohme e Investi Pharma. El Dr. Fabio Zambon es Gerente de Economía de la Salud en el Laboratorio Novo Nordisk. La Dra. Natalia Garrido Santos es Medical Scientific Liason del Laboratorio Novo Nordisk. La Dra. María Virginia Rodrí- guez es Asesora Médica del Laboratorio Novo Nordisk.

\section{INTRODUCCIÓN}

La enfermedad cardiovascular (infarto agudo de miocardio, accidente cerebrovascular y enfermedad arterial periférica) es la principal causa de morbimortalidad en pacientes con diabetes mellitus. Por este motivo se han estudiado los efectos cardiovasculares de las drogas antidiabéticas, en especial luego de la disposición de la Food and Drugs Administration (FDA) de los Estados Unidos de realizar estudios de seguridad cardiovascular de todos los nuevos agentes contra la diabetes ${ }^{1,2}$.

\section{Metformina}

\section{Mecanismo de acción}

\section{antihiperglucémico de la metformina}

- Estos efectos dependen de:

a) Reducción de la producción hepática de glucosa por disminución de gluconeogénesis y glucogenólisis.

b) Mejoría de la captación de glucosa muscular mediada por insulina.

- Estos efectos se asocian con:

a) Activación de AMPKinasa (adenosin mono fosfato kinasa, AMPK, incremento de la relación AMP(ATP), que genera desplazamiento desde acciones de consumo de energía (lipogénesis, gluconeogénesis) a acciones de ahorro de energía (captación de glucosa y oxidación lipídica).

b) Acción sobre el metabolismo mitocondrial (inhibición de fosforilación oxidativa, particularmente del complejo respiratorio I mitocondrial).

c) Inhibición de la acumulación de AMP cíclico (disminución de la actividad de la adenilciclasa e inhibición funcional del efecto del glucagón sobre la producción hepática de glucosa).

d) Incremento de la captación de glucosa intestinal por efecto sobre los transportadores de glucosa y cotransportadores sodio/glucosa 1, con incremento del metabolismo anaeróbico.

e) Mejoría de la secreción del péptido simil glucagón 1.

f) Modificación del microbioma intestinal: alteración del número de algunas especies microbianas intestinales, particularmente por incremento del número de bacterias que produce ácidos grasos de cadena corta ${ }^{3}$.

\section{Mecanismo de acción en la protección cardiovascular de la metformina}

Los mecanismos propuestos para la protección cardiovascular (CV) incluyen (Figura 1):

- Reducción del inhibidor del activador del plasminógeno 1 (PAI-1) y de la expresión de marcadores de activación del endotelio. El resultado es la mejoría de la función y respuesta vasodilatadora endotelial $\left.\right|^{3,4}$.

- Mejoría de los parámetros relacionados con la fibrinólisis ${ }^{5}$.

- Reducción de la hipersensibilidad plaquetaria ${ }^{5,6}$.

- Inhibición de los productos finales de glico- 
silación (en inglés, advanced glycation end products, AGEs) $)^{3,5}$.

- Prevención de los efectos de la angiotensina Il sobre el miocardio y los vasos?.

- Inhibición selectiva del estrés oxidativo3,8.

- Efectos pleiotrópicos mediados por vías bioquímicas como la respiración mitocondrial, activación de la AMPK, del óxido nítrico y el incremento intracelular de los niveles de adenosina². La activación de la AMPK es directa e indirecta a través de la inhibición de la fosforilación oxidativa del complejo respiratorio I. Esta acción es semejante a la producida por las condiciones de ayuno y ejercicio, influyendo en la captación de glucosa mediada por los GLUT-43,4,8.

- Efectos celulares antiaterogénicos a través de la inhibición de la conversión de monocitos a macrófagos, reducción de la infiltración de la pared vascular por los macrófagos, de la secreción de citoquinas inflamatorias y de la captación de colesterol por los macrófagos, con disminución de la formación de células espumosas ${ }^{3}$.

- Disminución del peso, redistribución del tejido adiposo central a depósitos subcutáneos y reducción del tejido adiposo epicárdico ${ }^{3,9}$.

- Mejoría del perfil lipídico 3 .

- Promoción del preacondicionamiento miocárdico ${ }^{10}$.

- Reducción de la apoptosis de los cardiomiocitos y adaptación metabólica de los mismos durante la isquemia, con desplazamiento de la oxidación de ácidos grasos libres a la utilización de glucosa ${ }^{8,10}$.

\section{Estudios de impacto cardiovascular de la metformina}

La evidencia que sugiere que la metformina mejora los resultados CV en pacientes con diabetes (DM) es sustancial, y se desprende de estudios randomizados y observacionales (Tabla 1).

La principal evidencia proviene del United Kingdom Prospective Diabetes Study (UKPDS) que demostró reducciones clínicas y estadísticamente significativas para los resultados finales para infarto de miocardio (IAM) y mortalidad por todas las causas $(-39 \%$ y $-36 \%$ respectivamente) en los pacientes tratados con metformina ${ }^{3,8,11-13}$.

El estudio Reducing with MetfOrmin Vascular Adverse Lesions in Type 1 Diabetes (REMOVAL) doble ciego, randomizado, controlado con placebo, que incluyó pacientes con diabetes tipo 1 (DM1), mostró una disminución significativa del engrosamiento máximo de la íntima media carotídea en los pacientes tratados con metformina e insulina ${ }^{14}$.

El Study on the Prognosis and Effect of Antidiabetic Drugs on Type 2 Diabetes Mellitus with Coronary Artery Disease (SPREAD-DIMCAD), multicéntrico, randomizado, controlado con placebo, reveló una reducción significativa de los eventos CV mayores comparada con glipizida ${ }^{15}$.

El estudio Hyperinsulinemia: the Outcome of its Metabolic Effect (HOME), randomizado, controlado contra placebo, en pacientes con DM2 tratados con insulina, mostró reducción de los resultados secundarios (IAM, insuficiencia cardíaca -IC-, síndrome coronario agudo, cambios electrocardiográficos, angioplastia transluminal, revascularización coronaria, ictus, accidente isquémico transitorio y enfermedad vascular periférica) en los pacientes tratados con metformina e insulina ${ }^{16}$.

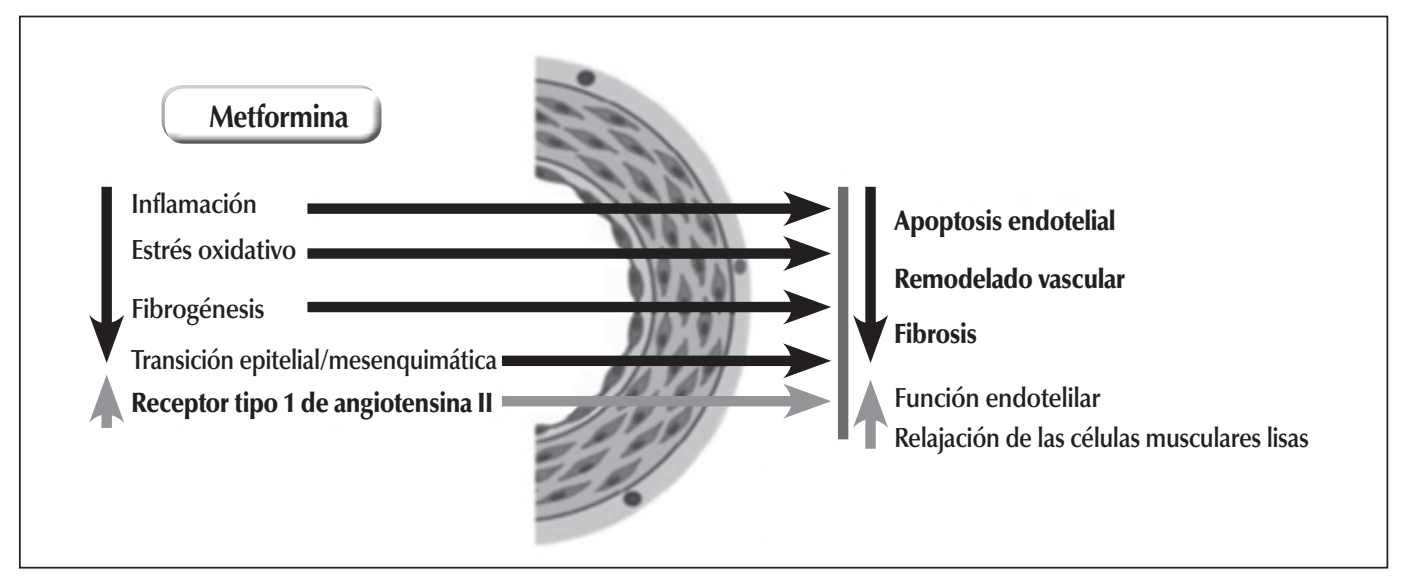

Fuente: Modificado de Nutr Metab Cardiovasc Dis 2017; 27(8):657-669.

Figura 1: Efectos de la metformina en el sistema vascular. 
Revista de la Sociedad Argentina de Diabetes Vol. 54 № 2 Suplemento I Jornadas Conjuntas del Comité de Graduados y el Comité de Hipertensión Arterial y otros FRCV. XVIII Jornadas del Comité de Graduados

Mayo-agosto de 2020: 107-122 ISSN 0325-5247 (impresa) ISSN 2346-9420 (en línea)

\begin{tabular}{|c|c|c|c|c|}
\hline Estudio & Seguimiento & Inclusión & Resultados & \\
\hline UKPDS $^{1}$ & 10,7 años & $\begin{array}{l}4.209 \text { individuos con DM2 } \\
25-65 \text { años }\end{array}$ & $\begin{array}{l}\text { Reducción del riesgo para: } \\
\text { Resultados finales relacionados } \\
\text { con DM (complicaciones macro y } \\
\text { microvasculares) } 32 \% \\
\text { Infarto de miocardio } 39 \% \\
\text { Ictus }\end{array}$ & 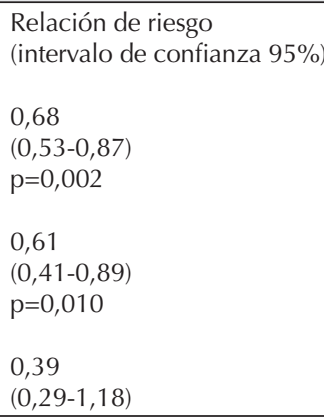 \\
\hline REMOVAL $^{2}$ & 4,2 años & $\begin{array}{l}493 \text { individuos con DM1 } \\
>40 \text { años }\end{array}$ & $\begin{array}{l}\text { Reducción del engrosamiento máximo } \\
\text { de íntima media carotídea (resultado final } \\
\text { intermedio de riesgo cardiovascular) }\end{array}$ & $\begin{array}{l}-0,013 \mathrm{~mm}(-0,024 \text { a }-0,03) \\
p=0,0093\end{array}$ \\
\hline $\begin{array}{l}\text { SPREAD- } \\
\text { DIMCAD }^{3}\end{array}$ & 5 años & $\begin{array}{l}304 \text { individuos con DM2 } \\
36-80 \text { años con EC }\end{array}$ & Reducción de MACE & $\begin{array}{l}\begin{array}{l}\text { Relación de riesgo } \\
\text { (intervalo de confianza 95\%) }\end{array} \\
0,54(0,30-0,90) \\
p=0,0026\end{array}$ \\
\hline $\mathrm{HOME}^{4}$ & 4,3 años & $\begin{array}{l}390 \text { individuos con DM2 } \\
\text { 30-80 años }\end{array}$ & $\begin{array}{l}\text { Reducción de los eventos } \\
\text { macrovasculares }\end{array}$ & $\begin{array}{l}\text { Relación de riesgo } \\
\text { (intervalo de confianza 95\%) } \\
0,61(0,40-0,94) \\
p=0,02\end{array}$ \\
\hline
\end{tabular}

EC: enfermedad coronaria; MACE: major adverse cardiovascular events leventos adversos cardiovasculares mayores: muerte cardiovascular, infarto de miocardio no falta, ictus no fatal).

${ }^{1}$ Lancet 1998; 352(9131):854-65 (contrarreferencia con cita 11).

2 Lancet Diabetes Endocrinol 2017; 5(8):597:609 (contrarreferencia con cita 14).

${ }^{3}$ Diabetes Care 2013; 36(5):1304-1311 (contrarreferencia con cita 15).

${ }^{4}$ Arch Intem Med 2009; 169 (6):616-626 (contrarreferencia con cita 16).

Tabla 1: Estudios que evalúan el impacto cardiovascular de la metformina.

\section{Sulfonilureas}

Aunque las sulfonilureas (SU) son los hipoglucemiantes orales más antiguos, su performance no ha sido evaluada mediante estudios de resultados CV (CVOT) específicos. De las disponibles en Argentina, glipizida y gliclazida son las SU pancreatoespecíficas, mientras que clorpropamida, glibenclamida y glimepirida presentan también afinidad por receptores extra pancreáticos. En 1970, tras el exceso de mortalidad reportado para tolbutamida, se estudió el vínculo entre las distintas SU y el preacondicionamiento isquémico. Glibenclamida obstaculizaría la cardioprotección y el fenómeno de warm up en contexto de isquemia, angioplastia, test con stress/esfuerzo, mientras que glimepirida parecería no interferir y gliclazida podría frenar el preacondicionamiento sólo a dosis supraterapéuticas y preservar parcialmente el warm up $p^{17-36}$.

Más allá del impacto sobre este mecanismo, no es claro que el control glucémico estricto mediante SU logre prevenir complicaciones macrovasculares, mortalidad CV o por cualquier causa. El Action to Control Cardiovascular Risk in Diabetes Study Group (ACCORD) fue discontinuado por aumento de mortalidad en la rama intensiva. Glimepirida, representante allí de las SU, no presentó reporte de ninguna particularidad vs otras drogas aunque el estudio no contaba con el diseño ni el poder suficiente para el testeo diferencial de los componentes terapéuticos. En el mismo sentido, grandes estudios experimentales no diseñados para evaluar eventos CV mayores (Major Adverse Cardiovascular Events, MACE), con un uso extensivo de SU, no reportaron diferencias en la seguridad de estas drogas en relación a otros grupos. Sin embargo, pocos son los ensayos controlados aleatorizados disponibles específicamente diseñados para evaluar la seguridad CV de SU. El control glucémico intensivo con gliclazida no mostró en Action in Diabetes and Vascular Disease: Preterax and Diamicron Modified Release Controlled EvaIuation (ADVANCE) efectos significativos sobre MACE y mortalidad, aún cuando las hipoglucemias severas en el grupo intensivo fueron mayores y se asociaron con un significativo aumento en MACE y muerte CV. Glimepirida, utilizada en el Cardiovascular Outcome Study of Linagliptin versus Glimepiride in Patients with Type 2 Diabetes 
(CAROLINA) como comparador activo para evaluar seguridad CV de linagliptina a largo plazo, no presentó diferencias en cuanto a endpoint primario (3P-MACE) o secundario (4P-MACE) a pesar de sí haber presentado mayor riesgo para todas las categorías de hipoglucemia, incluyendo las severas con requerimiento de hospitalización ${ }^{37-47}$.

Los metaanálisis que evalúan drogas antidiabéticas contemporáneas en relación a mortalidad global, CV o MACE presentan datos contradictorios, sin evidencia suficiente para arribar a conclusiones ${ }^{48-54}$.

Resulta conflictivo comparar las diversas SU entre sí. Estudios observacionales no hallarían diferencias significativas intraclase para MACE/mortalidad. De forma general podría decirse que glibenclamida presentaría la peor performance entre las SU actuales para: desarrollo de coronariopatía (también descripto para glipizida como tratamiento inicial), MACE en pacientes con y sin antecedentes, mortalidad CV y por cualquier causa (mayor a mayores dosis) con mayor riesgo hipoglucémico. Gliclazida podría ser la única SU asociable a riesgo menor, con menor mortalidad CV comparada con glibenclamida $y$, al igual que glimepirida, menores riesgos de desarrollo de coronariopatía/IAM, arritmias y complicaciones isquémicas en internación por IAM, así como mortalidad general ${ }^{55-75}$.

\section{Meglitinidas}

Las meglitinidas actualmente disponibles en Argentina son repaglinida y nateglinida. Los potenciales beneficios CV de las meglitinidas se relacionarían con acciones sobre el estado proaterogénico postprandial, y se reportan mejorías sobre marcadores inflamatorios y de función endotelial, parámetros de glicación, de stress oxidativo, de activación plaquetaria-coagulación, de parámetros lipídicos, homocisteína; incluso también se describen disminuciones significativas del espesor de la íntima media carotídea a lo largo de mediciones secuenciales con repaglinida ${ }^{76-83}$.

No hay a la fecha ensayos que hayan investigado de forma experimental específica meglitinidas en relación a MACE en la población con DM2. Lo más cercano a ello fue el Nateglinide and Valsartán in Impaired Glucosa Tolerance Outcomes Research (NAVIGATOR), estudio experimental que evaluó MACE en más de 9.000 individuos con factor de riesgo CV (FRCV) o enfermedad CV (ECV) manifiesta y tolerancia alterada a la glucosa (no DM). En este ensayo, nateglinida no demostró reducir la ocurrencia del combinado de mortalidad CV, IAM o accidente cerebrovascular (ACV) no fatales, hospitalización por IC o por angina inestable o revascularización arterial, así como tampoco previno la incidencia de DM2 aunque sí se asoció a mayor riesgo de hipoglucemias. En el Left Ventricular Dysfunction in Type 2 Diabetes Mellitus (DYDA), estudio prospectivo a dos años que investigó disfunción ventricular en 960 pacientes con DM2, repaglinida resultó factor de riesgo independiente para la ocurrencia del combinado mortalidad de cualquier causa y hospitalizaciones. Los autores cuestionaron si este resultado no se vinculaba a simple azar dado que no detectaron ni plausibilidad biológica ni mayor incidencia de eventos CV para repaglinida mediante análisis univariable ${ }^{84-86}$.

La información proveniente de grandes estudios poblacionales retrospectivos (con más de 20.000 pacientes en Suecia o toda la población adulta danesa con secretagogos) no muestra diferencias estadísticamente significativas en los riesgos de MACE para las meglitinidas (comparadas con otros antidiabéticos en el caso sueco y con metformina en el danés) ${ }^{74,87}$.

\section{Tiazolidinedionas}

Las tiazolidinedionas (TZDs) son antidiabéticos orales que aumentan la sensibilidad a la insulina al actuar sobre el tejido adiposo, muscular y hepático. Se unen a receptores nucleares activados por el proliferador de peroxisomas (PPAR). La rosiglitazona es un agonista PPAR-gamma puro, mientras que la pioglitazona también ejerce algunos efectos PPAR-alfa. Esto podría explicar sus diferentes efectos sobre lípidos y eventos $\mathrm{CV}$ isquémicos. Algunos metaanálisis de estudios con rosiglitazona en DM2 mostraron un mayor riesgo de IAM, mientras en otros no hubo aumento de IAM ni de mortalidad ${ }^{88-96}$.

A raíz de esto, la Food and Drug Administration (FDA, EE.UU.) cambió el marco regulatorio y exigió en adelante la realización de CVOT para la aprobación regulatoria. En el caso de rosiglitazona se efectuó el estudio Rosiglitazone Evaluated for Cardiovascular Outcomes in Oral Agent Combination Therapy for Type 2 Diabetes (RECORD). Luego de cinco años no hubo diferencias en el resultado primario (internación o muerte CV). El efecto en IAM fue no concluyente (HR 1,14; IC95\% 0,80$1,63)$, posiblemente afectado por un mayor uso de estatinas en el grupo de rosiglitazona, pero se duplicó el riesgo de $\mathrm{IC}^{97}$. 
Revista de la Sociedad Argentina de Diabetes Vol. 54 № 2 Suplemento I Jornadas Conjuntas del Comité de Graduados y el Comité de Hipertensión Arterial y otros FRCV. XVIII Jornadas del Comité de Graduados

Mayo-agosto de 2020: 107-122 ISSN 0325-5247 (impresa) ISSN 2346-9420 (en línea)

Posteriormente, la FDA llevó a cabo un estudio observacional, retrospectivo, sobre una base de datos de $>200.000$ jubilados, tratados con rosiglitazona o pioglitazona por tres años. Si bien el riesgo para IAM no fue significativo (IC95\% 0,96-1,18), sí aumentó el riesgo para ACV (HR 1,27), IC (HR 1,25), mortalidad global $(H R$ 1,14) y para el compuesto IAM, ACV, IC o mortalidad global con un HR de 1,18, un número necesario para dañar de 60 a lo largo de un año ${ }^{98,99}$.

El estudio Bypass Angioplasty Revascularization Investigation 2 Diabetes (BARI 2D) comparó distintas estrategias terapéuticas para enfermedad coronaria y control glucémico. Se cotejó tratamiento inicial con angioplastia o cirugía de bypass y sulfonilureas o insulina vs insulinosensibilizantes (metformina oTZDs). Después de un seguimiento promedio de 2,3 años, no hubo diferencia en el punto final primario (muerte) o el secundario principal (MACE compuesto: IAM, ACV o muerte CV) entre las distintas estrategias ${ }^{100}$.

El estudio PROspective pioglitAzone Clinical Trial In macroVascular Events (PROactive), con pioglitazona evaluó su efecto sobre MACE y mortalidad en 5.238 pacientes con ECV establecida. Se detuvo prematuramente por una disminución significativa en el endpoint secundario "principal": MACE de 3 puntos. Fue un estudio muy criticado desde el punto de vista metodológico ${ }^{101}$.

Insulin Resistance Intervention after Stroke (IRIS), el estudio más reciente de pioglitazona vs placebo, incluyó 3.876 pacientes con ACV o AIT recientes, sin $\mathrm{DM} 2$, pero con resistencia a la insulina (HOMA-IR $>3,0$ ). A los 4,8 años, el endpoint primario riesgo de ACV o IAM fue menor (9,0\% con pioglitazona vs $11,8 \%$ con placebo). Desarrolló DM2 3,8\% del grupo pioglitazona vs $7,7 \%$ con placebo (HR: 0,48; IC del $95 \%, 0,33$ a 0,69; $p<0,001)$. No hubo diferencias en mortalidad. Pioglitazona se asoció con aumento de peso, edemas y fracturas óseas ${ }^{102}$.

\section{Inhibidores de la dipeptidil peptidasa 4}

Uno de los grupos de fármacos recomendados en las guías de tratamiento de DM2 es el de los inhibidores de la dipeptidil peptidasa 4 (iDPP-4). Representan una estrategia terapéutica basada en incretinas que mejora el control de la glucosa mediante un mecanismo glucosa-dependiente ${ }^{103,104}$.

Con respecto a la evidencia que confiere seguridad CV a estas drogas, se han completado cinco grandes CVOT con iDPP-4, todos en pacientes con $\mathrm{DM} 2$, alto riesgo $\mathrm{CV}$ y seguimiento medio de 18 a 36 meses. Los estudios Saxagliptin Assessment of Vascular Outcomes Recorded in Patients with Diabetes Mellitus-Thrombolysis in Myocardial Infarction 53 (SAVOR-TIMI-53), Examination of Cardiovascular Outcomes with Alogliptin (EXAMINE) y Trial Evaluating Cardiovascular Outcomes with Sitagliptin (TECOS) demostraron que la adición de saxagliptina, alogliptina y sitagliptina respectivamente, no incrementó el riesgo de MACE en esta población en comparación con placebo, evaluados en forma prospectiva. Sin embargo, en el estudio SAVOR-TIMI-53 se describió que el uso de saxagliptina se asoció a un riesgo significativamente mayor de ingresos hospitalarios por IC (HR 1,27; 95\% IC 1,07-1,51). Asimismo, en un análisis post hoc del EXAMINE, se observó que un número mayor de pacientes con alogliptina fue hospitalizado por IC (HR 1,19, IC95\% 0,90-1,58) en comparación con el placebo. En TECOS, en cambio, no se observó un incremento de IC con sitagliptina ${ }^{105-107}$.

Por otra parte, estudios retrospectivos con uno a tres años de seguimiento no mostraron un mayor riesgo de hospitalización por IC con saxagliptina o sitagliptina. Más allá de esto, un metaanálisis describió una asociación entre el uso de iDPP-4 con un aumento del $13 \%$ de riesgo de IC $(R R=1,13 ; 95 \% \mid C=1,01-1,26)$, siendo similares resultados encontrados en un reciente estudio de cohorte vs SU (HR, 1,47; IC95\% 1,07-2,04). No está claro si el riesgo de IC es específico para ciertos iDPP-4 o es un efecto de clase para pacientes con ECV establecida, y si se extiende también a pacientes sin ECV, por lo que se necesita más evidencia para confirmar estos datos. Aunque el mecanismo que explica este mayor riesgo es desconocido, se postuló que la activación del sistema nervioso simpático podría implicar una lesión de los cardiomiocitos y su muerte. Por otro lado, se cree que mediante la inhibición de los DPP-4s se potenciaría la activación del factor 1 derivado del estroma, el neuropéptido $Y$ y la sustancia $P$ que, al estimular los receptores $\beta$-adrenérgicos, favorecería a la apoptosis de las células cardíacas ${ }^{108-112}$.

El estudio Cardiovascular and Renal Microvascular Outcome Study with Linagliptin in Patients with Type 2 Diabetes Mellitus (CARMELINA) mostró la seguridad CV de linagliptina al compararla con placebo, en asociación con terapéutica habitual en pacientes que presentaban enfermedad renal crónica (MACE: HR 1,02; IC del 95\%:0,89-1,17). En el estudio no se observó aumento del riesgo del compuesto renal de: muerte de causa renal, enferme- 
dad renal terminal o disminución sostenida $\geq 40 \%$ en la TFGe (tasa de filtrado glomerular) (HR 1,04; IC95\% 0,89-1,22), ni el riesgo de hospitalización por IC. El estudio CAROLINA, publicado recientemente, evaluó la seguridad CV de linagliptina en comparación con glimepirida. En pacientes con DM2 de corta evolución (seis años en promedio) y alto riesgo cardiovascular, linagliptina no incrementó el riesgo de MACE (HR 0,98 [IC 95,47\% 0,84-1,14] $\mathrm{p}<.001$ no inferioridad, $\mathrm{p}=0,76$ superioridad $)^{47,113,114}$.

Asimismo, con vildagliptina se estudió el riesgo de IC en el estudio The Vildagliptin in Ventricular Dysfunction Diabetes (VIVIDD) (con seguimiento a un año): 244 pacientes con DM2 e IC clase I-III, New York Heart Association (NYHA). No se encontraron diferencias significativas en la fracción de eyección del ventrículo izquierdo (VI) en comparación con placebo. Sin embargo, se evidenció aumento del volumen de fin de diástole $(17,06 \mathrm{ml} ; \mathrm{p}=0,007)$ y del volumen de fin de sístole del VI $(9,44 \mathrm{ml} ; p=0,06)$, así como del volumen sistólico $(p=0,002)$ sin cambios en el grosor de la pared del VI en la rama vildagliptina ${ }^{115}$.

Con teneligliptina, un estudio de farmacodinamia evaluó el intervalo QT y no se asoció con su prolongación con la dosis máxima de $40 \mathrm{mg}$. No obstante, concluyó que debe tenerse precaución al administrarse con medicamentos asociados con prolongación del QT. Actualmente se realiza un estudio que evaluará el efecto CV a largo plazo de teneligliptina, Teneligliptin on the Progressive Left Ventricular Diastolic Dysfunction With Type 2 Diabetes Mellitus (TOPLEVEL), cuyos resultados se esperan para 2023116,117.

Los datos de los CVOTs con iDPP-4 finalmen- te contrastan con los resultados de los análisis conjuntos previos y metaanálisis de estudios más pequeños que habían sugerido una reducción en el riesgo de MACE. Estas diferencias pueden explicarse fundamentalmente por los diseños de los estudios y las características de los pacientes ${ }^{118,119}$.

\section{Inhibidores del cotransportador sodio/glucosa 2}

\section{Mecanismo de acción}

La reabsorción renal de glucosa en el túbulo contorneado proximal (TCP) es mediada predominantemente (80-90\%) por los cotransportadores sodio/ glucosa 2 (SGLT-2, región proximal); la glucosa remanente es reabsorbida por los SGLT-1 (región distal). Los SGLT-2 son transportadores de alta capacidad y baja afinidad; los SGLT-1 son de baja capacidad y alta afinidad. En los pacientes con DM2 la expresión y actividad del SGLT-2 en elTCP están incrementadas, lo cual resulta en un aumento de la reabsorción renal de glucosa que contribuye a la hiperglucemia'20,121.

Los inhibidores de SGLT-2 (iSGLT-2) son antidiabéticos que disminuyen la glucemia (descenso de hemoglobina A1c - HbA1c- entre 0,4-1,1\%) a través de la reducción de la reabsorción renal de glucosa al promover glucosuria. Dado que su mecanismo de acción es independiente de insulina, no presentan riesgo para hipoglucemia. Los iSGLT-2 disponibles mundialmente son canaglifozina, dapaglifozina, empaglifozina, eruglifozina, ipraglifozina, luseoglifozina, sotaglifozina, remoglifozina y tofoglifozina ${ }^{120-124}$ (Figura 2).

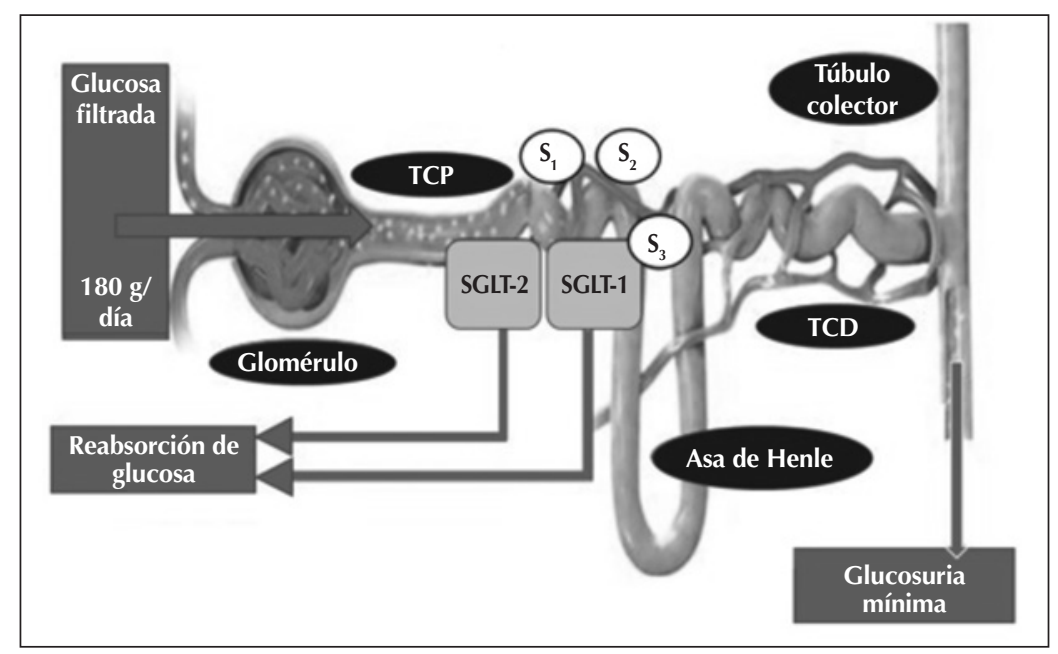

Fuente: Modificado de Freeman JS. Postgraduate Medicine 2013; 125 (3):214-226.

TCP: túbulo contorneado proximal; TCD: túbulo contorneado distal; SGLT: cotransportador sodio/glucosa; S: segmento del túbulo contorneado proximal.

Figura 2: Mecanismo de acción de los iSGLT-2. 


\section{Impacto cardiovascular}

El efecto protector CV de los iSGLT-2 es multifactorial e incluye efectos sistémicos (evidencia clínica) y efectos miocárdicos directos (evidencia preclínica). Entre los primeros se destacan los hemodinámicos como disminución de la resistencia vascular, de la presión arterial (sistólica de 4 a $6 \mathrm{mmHg}$ y diastólica de 1 a $2 \mathrm{mmHg}$ ), de la rigidez aórica, de la actividad del sistema nervioso simpático y de la precarga, todo relacionado con la natriuresis. Hay aumento de la diuresis osmótica. La respuesta metabólica está dada por un incremento de glucosuria, glucagón, ácidos grados libres, cuerpos cetónicos y angiotensina 1-7. En contraposición se observó reducción de la glucemia/insulinemia, HbA1c, glucotoxicidad, proteína $C$ reactiva ultra sensible, ácido úrico, peso, tejido adiposo, inflamación y disfunción endotelial. Los efectos en la homeostasis cardíaca incluyen reducción en la fibrosis y esteatosis miocárdica, en la inflamación cardíaca y vascular, y disminución en los MACE, además de un aumento en la función sisto-diastólica cardíaca.

A nivel miocárdico directo se puede detallar mejoría del consumo energético cardíaco por incremento de oxidación de cuerpos cetónicos (combustible de alta energía y oxigenación más eficiente, utilizado por el cardiomiocito de forma insulino-independiente), ácidos grasos libres, aminoácidos de cadena ramificada y lactato, y reducción de oxidación de glucosa, de la formación de AGE y acumulación de triglicéridos y glucosa. También disminución de la masa y diámetro VI, mejoría de la función diastólica del VI, reducción de rigidez arterial y de fibrosis miocárdica, y de activación neurohormonal (péptidos natriuréticos circulantes y catecolaminas) ${ }^{120,125-130 .}$

\section{Estudios de impacto cardiovascular}

Los CVOT sólo han sido completados con empagliflozina (Empagliflozin Cardiovascular Outco- me Event Trial in Type 2 Diabetes Mellitus Patients, EMPA-REG OUTCOME), dapagliflozina (Dapagliflozin Effect on Cardiovascular Events-Thrombolysis in Myocardial Infarction 58, DECLARE TIMI58) y canagliflozina (Canagliflozin Cardiovascular Assessment Study, CANVAS, y Canagliflozin and Renal Events in Diabetes with Established Nephropathy Clinical Evaluation, CREDENCE) (Tabla 2). Fueron estudios randomizados, doble ciego, comparados contra placebo. Empagliflozina y canagliflozina mostraron reducciones significativas de MACE. Dapagliflozina se asoció con reducción significativa de muerte CV y no fue inferior con respecto a MACE. Los tres estudios coinciden en el beneficio de estos fármacos en la disminución de la hospitalización por IC lo que correspondería a un efecto de clase. ${ }^{131-134}$.

Las diferencias en diseño y características de la población estudiada respecto de la presencia (o no) y tipo de ECV existente podría explicar algunas discrepancias en los resultados entre estudios, aunque no se descarta que la disimilitud se deba a peculiaridades intrínsecas a las moléculas. Dos metaanálisis compararon estas diferencias y arrojaron resultados contradictorios. Zelmiker destacó el beneficio sobre MACE en pacientes con ECV establecida (reducción del $11 \%$-IC 95\% 0,83-0,96) y Sinha sólo rescata el beneficio en la hospitalización por insuficiencia cardíaca y no muerte por ECV (MACE 0,83-IC95\% 0,66-1,03). Al momento de la revisión se encuentran en curso estudios con ertugliflozina (Cardiovascular Outcomes Following Ertugliflozin Treatment in Type 2 Diabetes Mellitus Participants With Vascular Disease, VERTIS-CV) ${ }^{135,136,137 .}$

La robustez de los resultados de seguridad cardiovascular y el impacto clínico de adicionar un iSGLT-2 al tratamiento estándar determinaron que las principales guías de manejo de DM2 ubiquen a estos fármacos entre las primeras opciones de tratamiento en pacientes con ECV establecida o alto/muy alto riesgo CV138,139. 
Revista de la Sociedad Argentina de Diabetes Vol. 54 № 2 Suplemento I Jornadas Conjuntas del Comité de Graduados y el Comité de Hipertensión Arterial y otros FRCV. XVIII Jornadas del Comité de Graduados Mayo-agosto de 2020: 107-122 ISSN 0325-5247 (impresa) ISSN 2346-9420 (en línea)

\begin{tabular}{|c|c|c|c|c|}
\hline Droga & $\begin{array}{l}\text { Estudio } \\
\text { (seguimiento) }\end{array}$ & Inclusión & Resultados & Relación de riesgo (IC95\%) \\
\hline Empagliflozina & $\begin{array}{l}\text { EMPA-REG } \\
\text { OUTCOME }^{1} \\
(3,1 \text { años })\end{array}$ & $\begin{array}{l}7.020 \text { individuos } \\
>18 \text { años con ECV } \\
\text { establecida (99\%) }\end{array}$ & $\begin{array}{l}\text { Reducción de MACE } 14 \% \\
\text { Reducción de muerte cardiovascular 38\% } \\
\text { Reducción mortalidad por todas las } \\
\text { causas } 32 \%\end{array}$ & $\begin{array}{l}\text { MACE } 0,86(0,74-0,99) \\
p=0,04\end{array}$ \\
\hline Dapagliflozina & $\begin{array}{l}\text { DECLARE } \\
\text { TIMI } 58^{2} \\
(4,2 \text { años })\end{array}$ & $\begin{array}{l}17.160 \text { individuos } \\
>40 \text { años con ECV } \\
\text { establecida ( } 41 \% \text { ) } \\
\geq 55 / 60 \text { años (hombre/ } \\
\text { mujer) con FRV }(59 \%)\end{array}$ & $\begin{array}{l}\text { Reducción de muerte cardiovascular vs. } \\
\text { placebo: } 4,9 \% \text { vs } 5,8 \%\end{array}$ & $\begin{array}{l}\text { MACE } 0,93(0,84-1,03) \\
p=0,17\end{array}$ \\
\hline \multirow[t]{2}{*}{ Canagloflozina } & $\begin{array}{l}\text { CANVAS }^{3} \\
(3,6 \text { años })\end{array}$ & $\begin{array}{l}10.142 \text { individuos } \\
\geq 30 \text { años con ECV }(66 \%) \\
>50 \text { años con FRCV }(34 \%)\end{array}$ & Reducción de MACE 14\% & $\begin{array}{l}\text { MACE } 0,86(0,75-0,97) \\
p=0,08\end{array}$ \\
\hline & $\begin{array}{l}\text { CREDENCE } \\
(2,6 \text { años })\end{array}$ & $\begin{array}{l}\text { 4,401 individuos } \\
49,6 \% \text { sin ECV } \\
50,4 \% \text { con } \mathrm{ECV}\end{array}$ & $\begin{array}{l}\text { Reducción de MACE 9,9\% } \\
\text { Reducción de muerte cardiovascular 5\% }\end{array}$ & $\begin{array}{l}\text { MACE } 0,80(0,67-0,95) \\
p=0,25\end{array}$ \\
\hline
\end{tabular}

ECV: enfermedad cardiovascular; FRCV: factores de riesgo vascular; MACE: major adverse cardiovascular events (eventos adversos cardiovasculares mayores: muerte cardiovascular, infarto de miocardio no falta, ictus no fatal).

${ }^{1}$ N Engl J Med 2015; 373(22):2117-2128.

${ }^{2}$ N Engl J Med 2019; 380(4):347-357.

${ }^{3}$ N Engl J Med 2017; 377 (7):644-657.

${ }^{4}$ Circulation 2019; 140(9):739:750.

Tabla 2: Estudios que evalúan el impacto cardiovascular de los inhibidores del cotransportador sodio/glucosa 2.

\section{Agonistas del receptor GLP-1}

Los análogos de GLP-1, exenatide y lixiexenatide, poseen una analogía del 53 y $50 \%$ respectivamente con el GLP-1 nativo. Por otro lado, los análogos liragutida, albiglutida, semaglutida y dulaglutida poseen una analogía del $90 \%$ o más con el GLP-1 nativo ${ }^{140}$.

El estudio Evaluation of LIXisenatide in Acute coronary syndrome (ELIXA), publicado en 2015, es el CVOT de lixisenatida que reveló no inferioridad de lixisenatida en el objetivo primario de MACE más hospitalización por angina inestable, pero no mostró superioridad, por lo cual se consideró un fármaco neutro a nivel CV ${ }^{141}$.

El estudio Exenatide Study of Cardiovascular Event Lowering (EXSCEL), publicado también en 2015, mostró que luego de 3,2 años de seguimiento, exenatida vs placebo no fue superior con respecto al objetivo primario (muerte CV, IAM o ACV). Sin embargo, la mortalidad por todas las causas fue menor en el grupo con exenatida ${ }^{142}$.

En el estudio Liraglutide and Cardiovascular Outcomes in Type 2 Diabetes (LEADER) el 81,3\% de la cohorte ya tenía ECV establecida y demostró que el objetivo compuesto primario MACE 3 puntos (IAM, ACV o muerte CV) ocurrió menos en los participantes en el grupo de tratamiento con liraglutide (13\%) que en el grupo placebo (14,9\%) (HR 0,87\%; IC del 95\%: 0,78 a 0,97; p,0,001 para no inferioridad; p5 0,01 para superioridad). Las muertes por causas CV se redujeron significativamente en el grupo de liraglutida $(4,7 \%)$ en comparación con el grupo placebo $(6,0 \%)$ (HR 0,78; IC95\% 0,66-0,93, p 5 0,007) ${ }^{143,144 .}$.

En el estudio Albiglutide and Cardiovascular Outcomes in Patients with Type 2 Diabetes and Cardiovascular Disease (HARMONY), 71\% de los pacientes tenía ECV, 25\% enfermedad arterial periférica, $25 \%$ enfermedad cerebrovascular y $20 \%$ IC. Se redujo el riesgo de muerte CV, IAM o ACV a una tasa de incidencia de 4,6 eventos por 100 años-persona en el grupo con albiglutida vs 5,9 eventos en el grupo placebo (proporción de HR 0,78, p 5 0,0006 para superioridad). Aunque demostró beneficios $\mathrm{CV}$, la molécula fue retirada del mercado por el promotor en $2018^{145}$.

En el estudio de seguridad cardiovascular Semaglutide Unabated Sustainability in Treatment of Type 2 Diabetes (SUSTAIN), el 83\% de los pacientes presentaba ECV establecida. El objetivo primario de no inferioridad frente a placebo para MACE 3 puntos se logró al demostrar incluso superioridad con un cociente de riesgo de 0,74: 108 pacientes (6,6\%) con semaglutida vs 146 pacientes $(8,9 \%)$ con placebo (HR 0,74; IC 95\% 0,58-0,95; p0,001) $)^{146}$.

En el estudio Peptide Inn OvatioN for Early DiabEtes TReatment (PIONEER) el 84,6\% de los pacientes tenía ECV establecida. Se confirmó la no inferioridad al placebo para el objetivo primario. El HR fue similar al estudio SUSTAIN-6, lo que podría 
sugerir que el efecto CV de la semaglutida es independiente de la ruta de administración. Aclara en la publicación, que muchos pacientes ya estaban medicados con iSGLT-2 lo que podría sumar efectos beneficiosos CV al resultado final147,148.

En el estudio Researching Cardiovascular Events with a Weekly Incretin in Diabetes (REWIND), a diferencia de los anteriores, sólo el 31\% de los participantes tenía ECV establecida. Durante una mediana de seguimiento de 5,4 años (RIC 5,1-5,9), el resultado compuesto primario ocurrió en 594 $(12,0 \%)$ participantes en el grupo de dulaglutida y en $663(13,4 \%)$ participantes en el grupo placebo (HR 0,88; IC 95\% 0,79-0,99; $p=0,026$ ). La mortalidad por todas las causas no fue diferente entre los grupos (536 [10,8\%] en el grupo de dulaglutida frente a $592[12,0 \%$ ] en el grupo placebo; HR 0,90; IC95\% 0,80-1,01; $p=0,067)^{149,150 .}$

\section{Insulina NPH y análogos de insulina de acción prolongada}

La ECV es la principal causa de muerte en pacientes con DM. La insulina podría jugar un rol importante tanto en la generación como en la progresión de la enfermedad ateroesclerótica, esto quizás ligado a la hiperinsulinemia. En estudios de experimentación se observaron lesiones ateroescleróticas en los animales tratados con insulina ${ }^{151}$.

La insulina isofánica o NPH (Neutral Protamine Hagedorn) y los análogos de insulina (glargina, detemir y degludec) difieren en sus características farmacocinéticas y farmacodinámicas, y los datos disponibles actualmente muestran que los análogos de insulina tienen un menor riesgo de episodios hipoglucemia que la insulina humana $\mathrm{NPH}$. Los resultados de estudios epidemiológicos y ensayos clínicos sugieren que la hipoglucemia podría tener un impacto negativo en la morbilidad y/o mortalidad CV152.

Nueve ensayos controlados aleatorizados compararon la eficacia entre insulina glargina (iGla) y $\mathrm{NPH}$. IGla se asoció con una mejor glucemia en ayunas en la mayoría de los estudios y la HbA1c mejoró ligeramente o no cambió, incluso se demostró una reducción de hipoglucemia nocturna ${ }^{152,153}$.

En una revisión de 14 estudios en DM1 y DM2, el tratamiento con insulina detemir (iDet) proporcionó un control glucémico similar o mejor, una menor variabilidad, una frecuencia similar o menor de hipoglucemia, y menor aumento de peso en comparación con la insulina NPH. En conclu- sión, los estudios identificados muestran que el uso de análogos de insulina de acción prolongada proporciona un control glucémico similar $\mathrm{O}$, a veces, superior en comparación con la NPH insulina, y puede asociarse con un menor riesgo de hipoglucemia, lo que podría traducirse en beneficios a nivel CV. No obstante, no existe suficiente evidencia de superioridad en lo que respecta a riesgo CV al comparar insulina NPH con los análogos de acción prolongada ${ }^{154}$.

Los análogos de acción prolongada se evaluaron en dos estudios de gran relevancia. El estudio Outcome Reduction with Initial Glargine Intervention (ORIGIN) comparó a la introducción temprana de iGla en el tratamiento de pacientes con DM2 o prediabetes con respecto a placebo en pacientes con riesgo $\mathrm{CV}$ aumentado. En los resultados del estudio ORIGIN se describe que el uso de la iGla no incrementaría la tasa de eventos CV al cabo de seis años de seguimiento en comparación con el placebo ${ }^{155}$.

El Estudio Trial Comparing Cardiovascular Safety of Insulin Degludec versus Insulin Glargine in Patients with Type 2 Diabetes at High Risk of Cardiovascular Events (DEVOTE), publicado en 2017, comparó el uso de la insulina degludec (iDeg) vs iGla en lo que respecta a la eficacia y seguridad $\mathrm{CV}$ teniendo en cuenta como objetivo primario combinado muerte $C V$, IAM no fatal y ACV no fatal. En el estudio 6.509 pacientes fueron asignados a recibir en forma randomizada iGla o iDeg. Al cabo de un seguimiento de dos años, no se observaron diferencias estadísticamente significativas en la tasa de eventos CV (tanto objetivo primario combinado como en los tres componentes del objetivo primario en forma separada) entre ambas ramas del estudio ${ }^{156}$.

\section{Análogos de insulina de acción rápida}

El objetivo primordial del control glucémico en pacientes con DM es retrasar la instauración de complicaciones crónicas, entre las que prevalece la ECV. La evidencia señala que la hiperglucemia postprandial se relacionaría con peores resultados CV. Los análogos de insulina de acción rápida (lispro, aspartica, glulisina) se asocian a menores excursiones de glucosa postprandial en personas con $\mathrm{DM}^{157}$.

Existen datos que muestran que insulina lispro (iLis) e insulina glulisina (iGlu) serían beneficiosas para el sistema vascular en comparación con insulina regular. Específicamente mejorarían el flujo sanguíneo postprandial microvascular en pacientes con DM1 ${ }^{158}$. 
En un estudio de cohorte retrospectivo se comparó el uso de análogos de insulina de acción rápida vs insulina regular humana en relación con la aparición de complicaciones diabéticas. Se excluyeron todos los pacientes que tenían ECV o recibían algún fármaco relacionado. Se encontraron 203 eventos macrovasculares $(22,2 \%)$ en el grupo de insulina regular humana y $147(10,7 \%)$ en el grupo de análogos de insulina de acción rápida, con una reducción significativa en las complicaciones macrovasculares asociadas con el uso de estos últimos ( $\mathrm{HR}=0,56$; IC del 95\%: 0,43-0,72). Entre los eventos macrovasculares, se observó una reducción significativa para cada condición clínica específica en los usuarios de análogos de insulina de acción rápida ${ }^{157}$.

Un estudio observacional retrospectivo, publicado en 2017 por Sevenson, comparó los tres análogos rápidos con el objetivo de evaluar la seguridad y mortalidad CV de pacientes con DM2. En el estudio no se advirtieron diferencias significativas en mortalidad, ECV, insuficiencia renal, hipoglucemia o hiperglucemia, aunque los autores describieron un efecto beneficioso de la insulina glulisina en la mortalidad general y en ACV, pero no observaron diferencias en términos de enfermedad coronaria o ECV fatal entre ninguno de los grupos. El riesgo de hiperglucemia severa fue mayor con aspártica que con iLis y el riesgo de hipoglucemia severa fue menor con iLis o iGlu (que con aspártica -iAsp), sobre todo para los pacientes mayores de 65 años y con insuficiencia renal ${ }^{159}$.

Se realizaron tres ensayos de seguridad y eficacia (18-26 semanas de tratamiento) con insulina aspártica rápida (Fiasp ${ }^{\circledR}$ ) vs insulina aspart (iAsp) en los que participaron 2.068 pacientes adultos aleatorizados con DM1 y DM2. Las variables principales se relacionaron con la eficacia comparativa de la insulina Fiasp y la seguridad en relación con eventos adversos a corto plazo. Más allá de que se describen eventos CV durante estos estudios, los autores no analizan la existencia de relación de los mismos con el uso de los fármacos y, por otra parte, no fueron estudios diseñados con el fin de evaluar impacto CV de la insulina Fiasp ${ }^{160,161,162 .}$

\section{CONCLUSIONES}

Desde que en 2007 un metaanálisis con rosiglitazona pusiera en tela de juicio la seguridad cardiovascular de las drogas antidiabéticas, se realizaron distintos estudios para demostrar la no inferioridad en este punto.
Para las drogas más antiguas no hay CVOTs, aunque metformina disminuyó el riesgo de IAM y mortalidad en el estudio UKPDS. A su vez, las sulfonilureas tendrían un resultado negativo en la cardioprotección. La de peor performance sería la glibenclamida. Mientras que gliclazida sería la más segura, glimepirida demostró no aumentar los MACE, sí en el reciente estudio CAROLINA. Las meglitinidas no aumentarían los MACE.

Después del citado metaanálisis, la rosigitazona no demostró aumento de IAM ni muerte en el estudio RECORD, aunque sí se asoció a mayor IC. La pioglitazona, en el estudio PROactive, disminuyó los MACE.

En cuanto a los inhibidores de la DPP-IV, todos mostraron resultados neutros aunque saxagliptina aumentó la hospitalización por IC y todavía no hay resultados para tenegliptina.

Los iSGLT-2 mostraron beneficios a nivel CV con alguna tendencia de clase. La empaglifozina tendría un mejor perfil. Algo similar sucede con los análogos de GLP-1 con demostrado beneficio CV, tanto en sus versiones subcutáneas, oral, diaria y semanal. Al referirnos a la insulina, son pocos los estudios que demuestran la seguridad CV para iGla, el ORIGIN, y para iDeg, el DEVOTE, mostraron no inferioridad en eventos $C V$, mientras que las ultra rápidas serían beneficiosas para el sistema vascular aunque deberían ampliarse los estudios.

\section{BIBLIOGRAFÍA}

1. American Diabetes Association. Cardiovascular disease and risk management: standards of medical care in diabetes. Diabetes Care 2020; 43(Suppl 1): S111-S134. https://doi.org/10.2337/ dc20-s010.

2. Dardano A, Miccoli R, Bianchi C, et al. Invited review. Series: Implications of the recent CVOTs in type 2 diabetes. Diabetes Research and Clinical Practice 2020;162: 108-112. https://doi. org/10.1016/j.diabres.2020.108112.

3. Zilov AV, Abdelaziz SI, Alshammary A, et al. Mechanisms of action of metformin with special reference to cardiovascular protection. Diabetes Metabolism Research and Reviews 2019; 35(7):1-12. https://doi.org/10.1002/dmrr.3173.

4. Varjabedian L, Bourji M, Pourafkari L, Nader ND. Cardioprotection by metformin: beneficial effects beyond glucose reduction. American Journal of Cardiovascular Drugs 2018; 18(3):181-193. https://doi.org/10.1007/s40256-018-0266-3.

5. Vague $\mathrm{P}$. Is metformin more than an oral hypoglycaemic agent? Diabetes \& Metabolism 2003; 29(4):6S5-6S7. https://doi. org/10.1016/s1262-3636(03)72782-7.

6. Grant PJ. Beneficial effects of metformin on haemostasis and vascular function in man. Diabetes \& Metabolism 2003; 29(4):6S44-6S52. https://doi.org/10.1016/s1262-3636(03)72787-6.

7. Nesti L, Natali A. Metformin effects on the heart and the cardiovascular system: a review of experimental and clinical data. Nutrition, Metabolism and Cardiovascular Diseases 2017; 27(8): 657-669. https://doi.org/10.1016/j.numecd.2017.04.009. 
Revista de la Sociedad Argentina de Diabetes Vol. 54 № 2 Suplemento I Jornadas Conjuntas del Comité de Graduados y el Comité de Hipertensión Arterial y otros FRCV. XVIII Jornadas del Comité de Graduados

Mayo-agosto de 2020: 107-122 ISSN 0325-5247 (impresa) ISSN 2346-9420 (en línea)

8. Scheen AJ, Paquot N. Metformin revisited: A critical review of the benefit-risk balance in at-risk patients with type 2 diabetes. Diabetes \& Metabolism 2013; 39(3):179-190. https://doi. org/10.1016/j.diabet.2013.02.006.

9. Ziyrek M, Kahraman S, Ozdemir E, Dogan A. Metformin monotherapy significantly decreases epicardial adipose tissue thickness in newly diagnosed type 2 diabetes patients. Revista Portuguesa de Cardiología 2019; 38(6):419-423. https://doi. org/10.1016/j.repc.2018.08.010.

10. Viollet B, Guigas B, García NS, Leclerc J, Foretz M, Andreelli F. Cellular and molecular mechanisms of metformin: an overview. Clinical Science 2011; 122(6):253-270. https://doi.org/10.1042/ cs20110386.

11. UK Prospective Diabetes Study (UKPDS) Group. Effect of intensive blood-glucose control with metformin on complications in overweight patients with type 2 diabetes (UKPDS 34). The Lancet 1998; 352(9131):854-865. https://doi.org/10.1016/s01406736(98)07037-8.

12. UK Prospective Diabetes Study Group. Tight blood pressure control and risk of macrovascular and microvascular complications in type 2 diabetes: UKPDS 38. BMJ 1998; 317(7160):703713. https://doi.org/10.1136/bmj.317.7160.703.

13. Schäfers RF. Do effects on blood pressure contribute to improved clinical outcomes with metformin? Diabetes \& Metabolism 2003; 29(4):6S62-6S70. https://doi.org/10.1016/s12623636(03)72789-x.

14. Petrie JR, Chaturvedi N, Ford I, et al. Cardiovascular and metabolic effects of metformin in patients with type 1 diabetes (REMOVAL): a double-blind, randomised, placebo-controlled trial. The Lancet Diabetes \& Endocrinology 2017; 5(8):597-609. https://doi.org/10.1016/s2213-8587(17)30194-8.

15. Hong J, ZhangY, Lai S, et al. Effects of metformin versus glipizide on cardiovascular outcomes in patients with type 2 diabetes and coronary artery disease. Diabetes Care 2012; 36(5):13041311. https://doi.org/10.2337/dc12-0719.

16. Kooy A, de Jager J, Lehert P, Bets D, Wulffelé MG, Donker AJM, Stehouwer CDA. Long-term effects of metformin on metabolism and microvascular and macrovascular disease in patients with type 2 diabetes mellitus. Archives of Internal Medicine 2009; 169(6):616. https://doi.org/10.1001/archinternmed.2009.20.

17. Serra-Sansone MP. Actualización en medicamentos antidiabéticos y riesgo cardiovascular. Rev Urug Cardiol 2016; 31:522-546.

18. Hinnen D, Krueger D. Cardiovascular risks in type 2 diabetes and the interpretation of cardiovascular outcome trials. Diabetes, Metabolic Syndrome and Obesity 2019;12 447-455.

19. Yandrapalli S, Jolly G, Horblitt A, et al. Cardiovascular benefits and safety of non-insulin medications used in the treatment of type 2 diabetes mellitus. Postgrad Med 2017 Nov; 129(8):811-821.

20. Cordiner R, Pearson E. Reflections on the sulphonylurea story: a drug class at risk of extinction or a drug class worth reviving? Diabetes Obes Metab 2019; 21:761-771.

21. Meinert $\mathrm{CL}$, Knatterud GL, ProutTE, et al. A study of the effects of hypoglycemic agents on vascular complications in patients with adult-onset diabetes: mortality results. Diabetes 1970; 19(Suppl):789-830.

22. Singh S, Bhat J, Wang PH. Cardiovascular effects of antidiabetic medications in type 2 diabetes mellitus. Curr Cardiol Rep 2013 Jan; 15(1):327-335.

23. Fisman EZ, Tenenbaum A. A cardiologic approach to non-insulin antidiabetic pharmacotherapy in patients with heart disease. Cardiovasc Diabetol 2009 Jul 20; 8:38.

24. Cleveland JC, Meldrum DR, Cain BS, et al. Oral sulfonylurea hypoglycemic agents prevent ischemic preconditioning in human myocardium: two paradoxes revisited. Circulation 1997; 96:29-32.

25. Tomai F, Crea F, Gaspardone A, et al. Ischemic preconditioning during coronary angioplasty is prevented by glibenclamide, a selective ATP-sensitive K + channel blocker. Circulation 1994; 90:700-5.
26. Klepzig H, Kober G, Matter C, et al. Sulfonylureas and ischaemic preconditioning: a double-blind placebo controlled evaluation of glimeperide and glibenclamide. Eur Heart J 1999; 20:439-46.

27. LeeTM, ChouTF. Impairment of myocardial protection in type 2 diabetic patients. J Clin Endocrinol Metab 2003; 88:531-537.

28. Scognamiglio R, Avogaro A, Vigili de Kreutzenberg $S$, et al. Effects of treatment with sulfonylurea drugs or insulin on ischemia-induced myocardial dysfunction in type 2 diabetes. Diabetes 2002; 51:808-812.

29. Tomai F, Danesi A, Ghini AS, et al. Effects of K(ATP) channel blockade by glibenclamide on the warm-up phenomenon. Eur Heart J 1999; 20:196-202.

30. Ovünç K. Effects of glibenclamide, a K(ATP) channel blocker, on warm-up phenomenon in type II diabetic patients with chronic stable angina pectoris. Clin Cardiol 2000; 23:535-539.

31. Ferreira BM, Moffa PJ, Falcão $A$, et al. The effects of glibenclamide, a K(ATP) channel blocker, on the warm-up phenomenon. Ann Noninvasive Electrocardiol 2005; 10:356-362.

32. Bilinska M, Potocka J, Korzeniowska-Kubacka I, et al. Warm-up phenomenon in diabetic patients with stable angina treated with diet or sulfonylureas. Coron Artery Dis 2007; 18:455-462.

33. Bogaty P, Kingma JG, Robitaille NM, et al. Attenuation of myocardial ischemia with repeated exercise in subjects with chronic stable angina: relation to myocardial contractility, intensity of exercise and the adenosine triphosphate-sensitive potassium channel. J Am Coll Cardiol 1998; 32:1665-1671.

34. Correa SD, Schaefer S. Blockade of K(ATP) channels with glibenclamide does not abolish preconditioning during demand ischemia. Am J Cardiol 1997; 79:75-78.

35. Rahmi Garcia RM, Rezende PC, Hueb W. Impact of hypoglycemic agents on myocardial ischemic preconditioning. World $\mathrm{J}$ Diabetes 2014; 5(3): 258-266 A.

36. Loubani M, Fowler A, Standen NB, et al. The effect of gliclazide and glibenclamide on preconditioning of the human myocardium. Eur J Pharmacol 2005; 515:142-149.

37. Panicker GK, Karnad DR, Salvi V, et al. Cardiovascular risk of oral antidiabetic drugs: current evidence and regulatory requirements for new drugs. J Assoc Physicians India 2012 Jan; 60:56-61.

38. Nunes AP, Iglay K, Radican L, et al. Hypoglycaemia seriousness and weight gain as determinants of cardiovascular disease outcomes among sulfonylurea users. Diabetes Obes Metab 2017 Oct; 19(10):1425-1435.

39. Patel A, MacMahon S, Chalmers J, et al; ADVANCE Collaborative Group. Intensive blood glucose control and vascular outcomes in patients with type 2 diabetes. N Engl J Med 2008; 358(24):2560-72.

40. Turnbull FM, Abraira C, et al; Control Group. Intensive glucose control and macrovascular outcomes in type 2 diabetes. Diabetologia 2009; 52:2288-98.

41. Gerstein HC, Miller ME, Byington RP, et al; Action to Control Cardiovascular Risk in Diabetes Study Group. Effects of intensive glucose lowering in type 2 diabetes. N Engl J Med 2008; 358:2545-2559.

42. Goff DC Jr, Gerstein HC, Ginsberg HN et al.; ACCORD Study Group. Prevention of cardiovascular disease in persons with type 2 diabetes mellitus: current knowledge and rationale for the Action to Control Cardiovascular Risk in Diabetes (ACCORD) trial. Am J Cardiol 2007; 99(12A):4i-20i.

43. UK Prospective Diabetes Study (UKPDS) Group. Intensive blood-glucose control with sulphonylureas or insulin compared with conventional treatment and risk of complications in patients with type 2 diabetes (UKPDS 33). Lancet 1998; 352(9131):837-53.

44. Viberti G, Kahn SE, Greene DA, et al. A diabetes outcome progression trial (ADOPT): an international multicenter study of the comparative efficacy of rosiglitazone, glyburide, and metformin in recently diagnosed type 2 diabetes. Diabetes Care 2002; 25:1737-1743. 
45. Paneni F, LüscherTF. Cardiovascular protection in the treatment of type 2 diabetes: a review of cinical trial results across drug classes. Am J Cardiol 2017 Jul 1; 120(1S):S17-S27.

46. Zoungas S, Patel A, Chalmers J, et al; ADVANCE Collaborative Group. Severe hypoglycemia and risks of vascular events and death. N Engl J Med 2010; 363:1410-1418.

47. Rosenstock J, Kahn SE, Johansen OE, et al. Effect of linagliptin vs glimepiride on major adverse cardiovascular outcomes in patients with type 2 diabetes: The CAROLINA Randomized Clinical Trial. JAMA 2019 Sep 19; 322(12):1155-1166. DOI: 10.1001/ jama.2019.13772.

48. Grenet G, Ribault S, Nguyen GB, et al. Glucose control safety $\&$ efficacy in type 2 diabetes, a systematic review and NETwork meta-analysis. PLoS One. 2019; 14(6):e0217701.

49. Varvaki-Rados D, Catan-Pinto L, Reck-Remonti L, et al. The association between sulfonylurea use and all-cause and cardiovascular mortality: a meta-analysis with trial sequential analysis of randomized clinical trials. PLoS Med 2016; 13:e1001992.

50. Hemmingsen B, Schroll JB, Lund SS, et al. Sulphonylurea monotherapy for patients with type 2 diabetes mellitus. Cochrane Database Syst Rev 2013 Apr 30; 4:CD009008.

51. Zhuang XD, He X, Yang DY, et al. Comparative cardiovascular outcomes in the era of novel anti-diabetic agents: a comprehensive network meta-analysis of 166,371 participants from 170 randomized controlled trials. Cardiovasc Diabetol 2018 Jun 5; 17(1):79.

52. Bain S, Druyts E, Balijepalli C, et al. Cardiovascular events and all-cause mortality associated with sulfonylureas compared with other antihyperglycaemic drugs: a Bayesian meta analysis of survival data. Diabetes Obes Metab 2017 Mar; 19(3):329-335.

53. Monami M, Genovese S, Mannucci E. Cardiovascular safety of sulfonylureas: a meta-analysis of randomized clinical trials. Diabetes Obes Metab 2013; 15(10):938-953.

54. Powell R, Christiansen C, Miller D. Meta-analysis of sulfonylurea therapy on long-term risk of mortality and cardiovascular events compared to other oral glucose-lowering treatments. Diabetes Ther 2018 Aug; 9(4):1431-1440.

55. Roumie CL, Hung AM, Greevy RA, et al. Comparative effectiveness of sulfonylurea and metformin monotherapy on cardiovascular events in type 2 diabetes mellitus: a cohort study. Ann Intern Med 2012 Nov 6; 157(9):601-610.

56. Douros A, Yin H, Yun Yu O, et al. Pharmacologic differences of sulfonylureas and the risk of adverse cardiovascular and hypoglycemic events. Diabetes Care 2017; 40:1506-1513.

57. Pantalone KM, Kattan MW, Yu C, et al. The risk of overall mortality in patients with type 2 diabetes receiving glipizide, glyburide, or glimepiride monotherapy: a retrospective analysis. Diabetes Care 2010 Jun; 33(6):1224-1229.

58. Pantalone $\mathrm{KM}$, et al. Increase in overall mortality risk in patients with type 2 diabetes receiving glipizide, glyburide or glimeperide monotherapy versus metformin: a retrospective analysis. Diabetes Obes Metab 2012; 14:803-9.

59. Simpson SH, Lee J, Choi S, Vandermeer B, Abdelmoneim AS, Featherstone TR. Mortality risk among sulfonylureas: a systematic review and network meta-analysis. Lancet Diabetes Endocrinol 2015; 3(1):43-51.

60. Evans JMM, Ogston SA, Reimann F, et al. No differences in mortality between users on pancreatic-specific and non-pancreatic specific sulfonylureas: a cohort analysis. Diabetes Obes Metab 2008; 10:350-2.

61. Andersson C, Gislason GH, Jorgensen $\mathrm{JH}$, et al. Comparable long term mortality risk associated with individual sulfonylureas in diabetes patients with heart failure. Diabetes Res Clin $\mathrm{Pr}$ 2011; 94:119-25.

62. Huang $Y$, Abdelmoneim AS, Light $P$, et al. Comparative cardiovascular safety of insulin secretagogues following hospitalization for ischemic heart disease among type 2 diabetes patients: a cohort study. J Diabetes Comp 2015; 29:196-202.
63. Juurlink DN, GomesT, Shah BR, Mamdani MM. Adverse cardiovascular events during treatment with glyburide (glibenclamide) or gliclazide in a high-risk population. DiabetMed 2012 Dec; 29(12):1524-1528.

64. Eriksson JW, Bodegard J, Nathanson D, et al. Sulphonylurea compared to DPP-4 inhibitors in combination with metformin carries increased risk of severe hypoglycemia, cardiovascular events, and all-cause mortality. Diabetes Res Clin Pract 2016 Jul; 117:39-47.

65. Monami M, Balzi D, Lamanna C, Barchielli A, et al. Are sulphonylureas all the same? a cohort study on cardiovascular and cancer-related mortality. Diabetes Metab Res Rev 2007; 23(6):479-84.

66. Abdelmoneim AS, Eurich DT, Senthilselvan A, et al. Dose-response relationship between sulfonylureas and major adverse cardiovascular events in elderly patients with type 2 diabetes. Pharmacoepidemiol Drug Saf 2016 Oct; 25(10):1186-1195.

67. Hung YC, Lin CC, Wang TY, Chang MP, Sung FC, Chen CC. Oral hypoglycaemic agents and the development of non-fatal cardiovascular events in patients with type 2 diabetes mellitus. Diabetes Metab Res Rev 2013; 29(8):673-9.

68. Sadikot S, Mogensen CE. Risk of coronary artery disease associated with initial sulfonylurea treatment of patients with type 2 diabetes: a matched case-control study. Diabetes Res Clin $\mathrm{Pr}$ 2008; 82:391-5.

69. Khalangot M, Tronko M, Kravchenko V, Kovtun V. Glibenclamide-related excess in total and cardiovascular mortality risks: data from large Ukrainian observational cohort study. Diabetes Res Clin Pract 2009; 86(3):247-53.

70. Simpson SH, Majumdar SR, Tsuyuki RT, Eurich DT, Johnson JA. Dose-response relation between sulfonylurea drugs and mortality in type 2 diabetes mellitus: a population-based cohort study. CMAJ 2006 Jan 17; 174(2):169-174.

71. Gangji AS, Cukierman T, Gerstein $\mathrm{HC}$, et al. A systematic review and meta-analysis of hypoglycemia and cardiovascular events. Diabetes Care 2007; 30:389-94.

72. Johnsen SP, Monster TB, Olsen ML, et al. Risk and short-term prognosis of myocardial infarction among users of antidiabetic drugs. Am JTher 2006; 13(2):134-40.

73. Zeller M, Danchin N, Simon D, et al; French Registry of Acute ST-Elevation and Non-ST-Elevation. Impact of type of preadmission sulfonylureas on mortality and cardiovascular outcomes in patients with diabetes with acute myocardial infarction. J Clin Endocrinol Metab 2010 Nov; 95(11): 4993-5002.

74. Schramm TK, Gislason GH, Vaag A, et al. Mortality and cardiovascular risk associated with different insulin secretagogues compared with metformin in type 2 diabetes, with or without a previous myocardial infarction: a nationwide study. Eur Heart J. 2011 Aug; 32(15):1900-1908. Erratum in: Eur Heart J 2012 May;33(10):1183.

75. Jørgensen $\mathrm{CH}$, Gislason GH, Andersson C, Ahlehoff O, Charlot M, Schramm TK, et al. Effects of oral glucose-lowering drugs on long term outcomes in patients with diabetes mellitus following myocardial infarction not treated with emergent percutaneous coronary intervention: a retrospective nationwide cohort study. Cardiovasc Diabetol 2010; 9:54.

76. Johansen $\mathrm{OE}$, Birkeland KI. Defining the role of repaglinide in the management of type 2 diabetes mellitus: a review. Am J Cardiovasc Drugs 2007; 7(5):319-335.

77. Sansone MP. Actualización en medicamentos antidiabéticos y riesgo cardiovascular. Rev Urug Cardiol 2016; 31:522-546.

78. Azimova K, San Juan Z, Mukherjee D. Cardiovascular safety profile of currently available diabetic drugs. Ochsner J 2014;14(4):616-632.

79. Derosa G, Mugellini A, Ciccarelli L, et al. Comparison between repaglinide and glimepiride in patients with type 2 diabetes mellitus: a one-year, randomized, double-blind assessment of metabolic parameters and cardiovascular risk factors. Clin Ther 2003 Feb; 25(2):472-84. 
Revista de la Sociedad Argentina de Diabetes Vol. 54 № 2 Suplemento I Jornadas Conjuntas del Comité de Graduados y el Comité de Hipertensión Arterial y otros FRCV. XVIII Jornadas del Comité de Graduados

Mayo-agosto de 2020: 107-122 ISSN 0325-5247 (impresa) ISSN 2346-9420 (en línea)

80. Esposito K, Giugliano D, Nappo F, et al. Regression of carotid atherosclerosis by control of postprandial hyperglycemia in type 2 diabetes mellitus. Circulation 2004 Jul 13; 110 (2):214-9.

81. Yngen M, Ostenson CG, Hjemdahl P, Wallén NH. Meal-induced platelet activation in type 2 diabetes mellitus: effects of treatment with repaglinide and glibenclamide. Diabet Med 2006 Feb; 23(2):134-40.

82. Manzella D, Grella R, Abbatecola AM, Paolisso G. Repaglinide administration improves brachial reactivity in type 2 diabetic patients. Diabetes Care 2005 Feb; 28(2): 366-371.

83. Rizzo MR, Barbieri M, Grella R, et al. Repaglinide has more beneficial effect on cardiovascular risk factors than glimepiride: data from meal-test study. Diabetes Metab 2005 Jun; 31(3 Pt 1):255-60.

84. Scheen AJ. NAVIGATOR: a trial of prevention of cardiovascular complications and type 2 diabetes with valsartan and/or nateglinide. Rev Med Liege 2010 Apr; 65(4): 217-223.

85. Holman RR, Haffner SM, McMurray JJ, et al; NAVIGATOR Study Group. Effect of nateglinide on the incidence of diabetes and cardiovascular events. N Engl J Med 2010 Apr 22; 362(16):14631476. Erratum in: N Engl J Med 2010 May 6; 362(18):1748.

86. Cioffi G, Faggiano P, Lucci D, et al. Left ventricular dysfunction and outcome at two-year follow-up in patients with type 2 diabetes: The DYDA study. Diabetes Res Clin Pract 2013 Aug; 101(2):236-242.

87. Ekström N, Svensson AM, Miftaraj M, et al. Cardiovascular safety of glucose-lowering agents as add-on medication to metformin treatment in type 2 diabetes: report from the Swedish National Diabetes Register. Diabetes Obes Metab 2016 Oct; 18(10):990-8.

88. Yki-Järvinen H. Thiazolidinediones. N Engl J Med 2004; 351:1106.

89. Vidal-Puig AJ, Considine RV, Jimenez-Liñan M, et al. Peroxisome proliferator-activated receptor gene expression in human tissues. Effects of obesity, weight loss, and regulation by insulin and glucocorticoids. J Clin Invest 1997; 99:2416.

90. Nissen SE, Wolski K. Effect of rosiglitazone on the risk of myocardial infarction and death from cardiovascular causes. N Engl J Med 2007; 356:2457.

91. Rosen CJ. The rosiglitazone story-lessons from an FDA Advisory Committee meeting. N Engl J Med 2007; 357:844.

92. Singh S, Loke YK, Furberg CD. Long-term risk of cardiovascular events with rosiglitazone: a meta-analysis. JAMA 2007; 298:1189.

93. Nissen SE, Wolski K. Rosiglitazone revisited: an updated metaanalysis of risk for myocardial infarction and cardiovascular mortality. Arch Intern Med 2010; 170:1191.

94. Cobitz A, Zambanini A, Sowell M, et al. A retrospective evaluation of congestive heart failure and myocardial ischemia events in 14,237 patients with type 2 diabetes mellitus enrolled in 42 short-term, double-blind, randomized clinical studies with rosiglitazone. Pharmacoepidemiol Drug Saf 2008; 17:769.

95. Richter B, Bandeira-Echtler E, Bergerhoff K, et al. Rosiglitazone for type 2 diabetes mellitus. Cochrane Database Syst Rev 2007; CD006063.

96. Diamond GA, Bax L, Kaul S. Uncertain effects of rosiglitazone on the risk for myocardial infarction and cardiovascular death. Ann Intern Med 2007; 147:578.

97. Home PD, Pocock SJ, Beck-Nielsen $\mathrm{H}$, et al. Rosiglitazone evaluated for cardiovascular outcomes in oral agent combination therapy for type 2 diabetes (RECORD): a multicentre, randomised, open-label trial. Lancet 2009; 373:2125.

98. Goldberg RB, Kendall DM, Deeg MA, et al. A comparison of lipid and glycemic effects of pioglitazone and rosiglitazone in patients with type 2 diabetes and dyslipidemia. Diabetes Care 2005; 28:1547.

99. Graham DJ, Ouellet-Hellstrom R, MaCurdy TE, Ali F, Sholley C, Worrall C, Kelman JA. Risk of acute myocardial infarction, stroke, heart failure, and death in elderly medicare patients treated wth rosiglitazone or pioglitazone. JAMA 2010; 304(4):411. https://doi.org/10.1001/jama.2010.920.
100. The BARI 2D Study Group. A randomized trial of therapies for type 2 diabetes and coronary artery disease. New England Journal of Medicine 2009; 360(24):2503-2515. https://doi. org/10.1056/nejmoa0805796.

101. Dormandy JA, Charbonnel B, Eckland DJ, et al. Secondary prevention of macrovascular events in patients with type 2 diabetes in the PROactive Study (PROspective pioglitAzone Clinical Trial In macroVascular Events): a randomised controlled trial. Lancet 2005; 366:1279.

102. Inzucchi SE, Furie KL. The IRIS (Insulin Resistance Intervention after Stroke) trial: A new perspective on pioglitazone. Journal of Diabetes 2016; 8(5):607-609. https://doi.org/10.1111/17530407.12399 .

103. American Diabetes Association. Pharmacologic approaches to glycemic treatment: standards of medical care in diabetes 2020. Diabetes Care 2019; 43(Suppl 1): S98-S110. https://doi. org/10.2337/dc20-s009.

104. Vella A. Mechanism of action of DPP-4 inhibitors new insights. The Journal of Clinical Endocrinology \& Metabolism 2012; 97(8):2626-2628. https://doi.org/10.1210/jc.2012-2396.

105. Scirica BM, Bhatt DL, Braunwald E, et al. Saxagliptin and cardiovascular outcomes in patients with type 2 diabetes mellitus. $\mathrm{N}$ Engl J Med 2013; 369:1317-26.

106. White WB, Cannon CP, Heller SR, et al. Alogliptin after acute coronary syndrome in patients with type 2 diabetes. $N$ Engl $\mathrm{J}$ Med 2013; 369:1327-35.

107. Green JB, Bethel MA, Armstrong PW, et al. Effect of sitagliptin on cardiovascular outcomes in type 2 diabetes. N Engl J Med $2015 ; 373: 232-42$.

108. Ou SM, Shih CJ, Chao PW, et al. Effects on clinical outcomes of adding dipeptidyl peptidase- 4 inhibitors vs sulfonylureas to metformin therapy in patients with type 2 diabetes mellitus. Ann Intern Med 2015; 163(9):663.

109. Toh S, Hampp C, Reichman ME, et al. Risk for hospitalized heart failure among new users of saxagliptin, sitagliptin, and other antihyperglycemic drugs: a retrospective cohort Sstudy. Ann Intern Med 2016; 164(11):705.

110. Rehman MB, Tudrej BV, Soustre J, et al. Efficacy and safety of DPP-4 inhibitors in patients with type 2 diabetes: Meta-analysis of placebo-controlled randomized clinical trials. Diabetes $\mathrm{Me}-$ tab 2017 Feb; 43(1):48-58.

111. Kim KJ, Choi J, Lee J. Dipeptidyl peptidase-4 inhibitor compared with sulfonylurea in combination with metformin: cardiovascular and renal outcomes in a propensity-matched cohort study. Cardiovasc Diabetol 2019; 18:28.

112. Packer M. Do DPP-4 inhibitors cause heart failure events by promoting adrenergically mediated cardiotoxicity? Clues from laboratory models and clinical trials. Circ Res 2018; 122:928-32.

113. Rosenstock J, Perkovic V, Johansen OE, et al. Effect of linagliptin vs placebo on major cardiovascular events in adults with type 2 diabetes and high cardiovascular and renal risk: the CARMELINA randomized clinical trial. JAMA 2019; 321:69-79.

114. Marx N, Rosenstock J, Kahn SE, et al. Design and baseline characteristics of the CARdiovascular outcome trial of LINAgliptin versus glimepiride in type 2 diabetes (CAROLINA ${ }^{\oplus}$ ). Diab Vasc Dis Res 2015; 12:164-74.

115. McMurray JJ, Ponikowksi P, Bolli GB, et al. Vildagliptin in Ventricular Dysfunction Diabetes Trial (VIVIDD). Eur J Heart Fail 2013; 12(suppl):1.

116. Kishimoto M. Teneligliptin: a DPP-4 inhibitor for the treatment of type 2 diabetes. Diabetes Metab Syndr Obes Targets Ther 2013; 6:187-95.

117. National cerebral and cardiovascular center. Teneligliptin on the progressive left ventricular diastolic dysfunction with type 2 diabetes mellitus study. clinicaltrials.gov NCT02449330. https:// clinicaltrials.gov/ct2/show/NCT02449330?term=TOPLEVEL\&ra $n k=1$. Acceso: 14/19/2019. 
118. Mannucci E, Mosenzon O, Avogaro A. Analyses of results from cardiovascular safety trials with DPP-4 inhibitors: cardiovascular outcomes, predefined safety outcomes, and pooled analysis and meta-analysis. Diabetes Care 2016; 39(Suppl 2):S196-204.

119. Monami M, Ahren B, Dicembrini I, Mannucci E. Dipeptidyl peptidase-4. inhibitors and cardiovascular risk: a meta-analysis of randomized clinical trials. Diabetes Obes Metab 2013; 15:112-20.

120. Chilton RJ. Effects of sodium-glucose cotransporter-2 inhibitors on the cardiovascular and renal complications of type 2 diabetes. Diabetes, Obesity and Metabolism 2019; 22(1):16-29. https://doi.org/10.1111/dom.13854.

121. Giugliano D, Esposito K. Class effect for SGLT-2 inhibitors: a tale of 9 drugs. Cardiovascular Diabetology 2019; 18(1):1-4. https:// doi.org/10.1186/s12933-019-0899-9.

122. John M, GopinathD, Jagesh R. Sodium-glucose cotransporter 2 inhibitors with insulin in type 2 diabetes: clinical perspectives. Indian Journal of Endocrinology and Metabolism 2016; 20(1):22-31. https://doi.org/10.4103/2230-8210.172268.

123. Thomas MC, Cherney DZI.The actions of SGLT2 inhibitors on metabolism, renal function and blood pressure. Diabetologia 2018; 61(10):2098-2107. https://doi.org/10.1007/s00125-018-4669-0.

124. Lupsa BC, Inzucchi SE. Use of SGLT2 inhibitors in type 2 diabetes: weighing the risks and benefits. Diabetologia 2018; 61(10):2118-2125. https://doi.org/10.1007/s00125-018-4663-6.

125. Vardeny $\mathrm{O}$, Vaduganathan $\mathrm{M}$. Practical guide to prescribing sodium-glucose cotransporter 2 inhibitors for cardiologists. JACC Heart Failure 2019; 7(2):169-172. https://doi.org/10.1016/j. jchf.2018.11.013.

126. Evans M, Morgan AR, Yousef Z. What next after metformin? Thinking beyond glycaemia: are SGLT-2 ilnhibitors the answer? Diabetes Therapy 2019; 10(5):1719-1731. https://doi.org/10.1007/ s13300-019-00678-z.

127. Lehrke M. SGLT-2 Inhibition. Journal of the American College of Cardiology 2019; 73(15)-1945-1947. https://doi.org/10.1016/j. jacc.2019.02.023.

128. Kaplan A, Abidi E, El-Yazbi A, et al. Direct cardiovascular impact of SGLT-2 inhibitors: mechanisms and effects. Heart Failure Reviews 2018; 23(3):419-437. https://doi.org/10.1007/s10741-0179665-9.

129. Garcia-Ropero A, Santos-Gallego CG, Zafar MU, Badimon JJ. Metabolism of the failing heart and the impact of SGLT-2 inhibitors. Expert Opinion on Drug Metabolism \& Toxicology 2019; 15(4):275-285. https://doi.org/10.1080/17425255.2019.1588886.

130. Verma S, McMurray JJV. SGLT-2 inhibitors and mechanisms of cardiovascular benefit: a state-of-the-art review. Diabetologia 2018; 61(10):2108-2117. https://doi.org/10.1007/s00125-018-4670-7.

131. Zinman B, Wanner C, Lachin JM, et al. Empagliflozin, cardiovascular outcomes, and mortality in type 2 diabetes. New England Journal of Medicine 2015; 373(22):2117-2128. https:// doi.org/10.1056/nejmoa1504720.

132. Wiviott SD, Raz I, Bonaca MP, et al. Dapagliflozin and cardiovascular outcomes in type 2 diabetes. New England Journal of Medicine 2019; 380(4):347-357. https://doi.org/10.1056/nejmoa1812389.

133. Neal B, Perkovic V, Mahaffey KW, et al. Canagliflozin and cardiovascular and renal events in type 2 diabetes. New England Journal of Medicine 2017; 377(7):644-657. https://doi.org/10.1056/nejmoa1611925.

134. Mahaffey KW, Jardine MJ, Bompoint S, et al. Canagliflozin and cardiovascular and renal outcomes in type 2 diabetes mellitus and chronic kidney disease in primary and secondary cardiovascular prevention groups. Circulation 2019; 140(9):739-750. https://doi.org/10.1161/circulationaha.119.042007.

135. Gallwitz B. The cardiovascular benefits associated with the use of sodium-glucose cotransporter 2 inhibitors. Real-world data. European Endocrinology 2018; 14(1):17-23. https://doi. org/10.17925/ee.2018.14.1.17.
136. ZelnikerTA, Wiviott SD, Raz I, et al. SGLT-2 inhibitors for primary and secondary prevention of cardiovascular and renal outcomes in type 2 diabetes: a systematic review and meta-analysis of cardiovascular outcome trials. The Lancet 2019; 393(10166):31-39. https://doi.org/10.1016/s0140-6736(18)32590-x.

137. Sinha B, Ghosal S. Sodium-glucose cotransporter-2 inhibitors (SGLT-2i) reduce hospitalization for heart failure only and have no effect on atherosclerotic cardiovascular events: a metaAnalysis. Diabetes Therapy 2019; 10(3):891-899. https://doi. org/10.1007/s13300-019-0597-3.

138. Cavallari I, Maddaloni E. Cardiovascular effects of SGLT-2 inhibitors: What we have learned from cardiovascular outcome trials and what we still need to understand. Diabetes/Metabolism Research and Reviews 2019; 35(4):e3124. https://doi. org/10.1002/dmrr.3124.

139. Cosentino F, Grant PJ, Aboyans V, et al. 2019 ESC Guidelines on diabetes, pre-diabetes, and cardiovascular diseases developed in collaboration with the EASD. European Heart Journal 2019; 41(2):255-323. https://doi.org/10.1093/eurheartj/ehz486.

140. Aroda VR. A review of GLP-1 receptor agonists: evolution and advancement, through the lens of randomised controlled trials. Diabetes, Obesity and Metabolism 2018; 20:22-33. https://doi. org/10.1111/dom.13162.

141. Pfeffer MA, Claggett $B$, Diaz R, et al. Lixisenatide in patients with type 2 diabetes and acute coronary syndrome. New England Journal of Medicine 2015; 373(23):2247-2257. https:// doi.org/10.1056/nejmoa1509225.

142. Mentz RJ, Bethel MA, Merrill P, et al. Effect of once-weekly exenatide on clinical outcomes according to baseline risk in patients with type 2 diabetes mellitus: insights from the EXSCEL trial. Journal of the American Heart Association 2018; 7(19):126. https://doi.org/10.1161/jaha.118.009304.

143. American Diabetes Association. Cardiovascular disease and risk management: standards of medical care in diabetes-2019. Diabetes Care 2018; 42(Supplement 1):S103-S123. https://doi. org/10.2337/dc19-s010.

144. Marso SP, Daniels GH, Brown-Frandsen K, et al. Liraglutide and cardiovascular outcomes in type 2 diabetes. New England Journal of Medicine 2016; 375(4):311-322. https://doi.org/10.1056/nejmoa1603827.

145. Hernández AF, Green JB, Janmohamed S, et al. Albiglutide and cardiovascular outcomes in patients with type 2 diabetes and cardiovascular disease (Harmony Outcomes): a double-blind, randomised placebo-controlled trial. Lancet. 2018; 392:1519-1529.

146. Marso SP, Bain SC, Consoli $A$, et al. Semaglutide and cardiovascular outcomes in patients with type 2 diabetes. New England Journal of Medicine 2016; 375(19):1834-1844. https:// doi.org/10.1056/nejmoa1607141.

147. Bain SC, Mosenzon O, Arechavaleta R, et al. Cardiovascular safety of oral semaglutide in patients with type 2 diabetes: Rationale, design and patient baseline characteristics for the PIONEER 6 trial. Diabetes, Obesity and Metabolism 2018; 21(3):499508. https://doi.org/10.1111/dom.13553.

148. Husain M, Birkenfeld AL, Donsmark M, Dungan K, et al. Oral semaglutide and cardiovascular outcomes in patients with type 2 diabetes. New England Journal of Medicine 2019; 381(9):841851. https://doi.org/10.1056/nejmoa1901118.

149. Gerstein HC, Colhoun HM, Dagenais GR, et al. Design and baseline characteristics of participants in the researching cardiovascular events with a weekly incretin in diabetes (REWIND) trial on the cardiovascular effects of dulaglutide. Diabetes, Obesity and Metabolism 2017; 20(1):42-49. https://doi.org/10.1111/dom.13028.

150. Gerstein HC, Colhoun HM, Dagenais GR, et al. Dulaglutide and cardiovascular outcomes in type 2 diabetes (REWIND): a double-blind, randomised placebo-controlled trial. Lancet 13 2019;394(10193):121-130

151. Donner T, Joseph J. Long-term insulin glargine therapy in type 2 diabetes mellitus: a focus on cardiovascular outcomes. Vascular Health and Risk Management 2015; 107. https://doi. org/10.2147/vhrm.s50286. 
Revista de la Sociedad Argentina de Diabetes Vol. 54 № 2 Suplemento I Jornadas Conjuntas del Comité de Graduados y el Comité de Hipertensión Arterial y otros FRCV. XVIII Jornadas del Comité de Graduados

Mayo-agosto de 2020: 107-122 ISSN 0325-5247 (impresa) ISSN 2346-9420 (en línea)

152. Laranjeira FO, de Andrade KRC, Figueiredo ACMG, et al. Longacting insulin analogues for type 1 diabetes: an overview of systematic reviews and meta-analysis of randomized controlled trials. PLOS ONE 2018; 13(4):e0194801. https://doi.org/10.1371/ journal.pone.0194801.

153. Mannucci E, Giannini S, Dicembrini I. Cardiovascular effects of basal insulins. Drug, Healthcare and Patient Safety 2015; 113. https://doi.org/10.2147/dhps.s43300.

154. Frier BM, Russell-Jones D, Heise T. A comparison of insulin detemir and neutral protamine Hagedorn (isophane) insulin in the treatment of diabetes: a systematic review. Diabetes, Obesity and Metabolism 2013; 15(11):978-986. https://doi.org/10.1111/ dom.12106.

155. Gerstein HC, Bosch J, Dagenais GR, et al. Basal insulin and cardiovascular and other outcomes in dysglycemia. New England Journal of Medicine 2012; 367(4):319-328. https://doi. org/10.1056/nejmoa1203858.

156. Marso SP, McGuire DK, Zinman B, et al. Efficacy and safety of degludec versus glargine in type 2 diabetes. New England Journal of Medicine 2017; 377(8):723-732. https://doi.org/10.1056/nejmoa1615692.

157. Cammarota S, Falconio LM, Bruzzese D, et al. Lower rate of cardiovascular complications in patients on bolus insulin analogues: a retrospective population-based cohort study. PLoS ONE 2013; 8(11):e79762. https://doi.org/10.1371/journal. pone.0079762.
158. Raskin P, Fordan S. Vascular effects of rapid-acting insulin analogs in the diabetic patient: a review. Vascular Health and Risk Management 2009; 225. https://doi.org/10.2147/vhrm.s3402.

159. Svensson AM, Miftaraj M, Franzén S, Eliasson B. Clinical effects, cardiovascular and renal outcomes associated with rapid-acting insulin analogs among individuals with type 2 diabetes: a nation-wide observational cohort study. Clinical Diabetes and Endocrinology 2017; 3(1):1-8. https://doi.org/10.1186/ s40842-017-0043-2.

160. Russell-Jones D, Bode BW, De Block C, et al. Fast-acting insulin aspart improves glycemic control in basal-bolus treatment for type 1 diabetes: results of a 26-week multicenter, active-controlled, treat-to-target, randomized, parallel-group trial (onset 1). Diabetes Care 2017; 40(7):943-950. https://doi.org/10.2337/ dc16-1771.

161. Bowering $\mathrm{K}$, Case $\mathrm{C}$, Harvey J, et al. Faster aspart vs insulin aspart as part of a basal-bolus regimen in inadequately controlled type 2 diabetes: the onset 2 trial. Diabetes Care 2017; 40(7):951957. https://doi.org/10.2337/dc16-1770.

162. Rodbard HW, Tripathy D, Vidrio-Velázquez M, et al. Adding fastacting insulin aspart to basal insulin significantly improved glycaemic control in patients with type 2 diabetes: A randomized, 18-week, open-label, phase 3 trial (onset 3). Diabetes, Obesity and Metabolism 2017; 19(10):1389-1396. https://doi. org/10.1111/dom.12955. 